\title{
Specialization by Practice Location
}

D epending on one's perspective, the current expansion of hospitalism can be viewed as either an advance or an anachronism. Those who believe it is an advance argue that this new breed of physician can provide greater expertise and efficiency than traditional members of a hospital medical staff. ${ }^{1}$ Those who believe it is an anachronism see merely the re-creation of the previously discarded "house physician" and predict that the same problems will recur and lead to the hospitalist's demise. These problems include unacceptable work schedules, failure to integrate care with physicians in the community, and inadequate continuity of care.

Like it or not, however, hospitalists seem to be here to stay, or rather back here to stay, because hospitals want and need them. In facilities in which lengths of stay are relatively long by present standards, the introduction of hospitalists appears to shorten stays and reduce costs. ${ }^{2,3}$ Moreover, hospital administrators find it far easier to deal with a small group of physician employees whose efforts are dedicated solely to their institution than with a heterogeneous group of office-based practitioners who come to the hospital only when necessary. When it comes to complying with onerous documentation requirements and following clinical practice guidelines, the difference between these two groups is like the difference between dogs and cats-one group is easier to train than the other. Some proponents of hospitalism have argued that dedicated inpatient physicians provide higher-quality care than generalists, but no data have emerged yet to confirm or refute this contention. On the other side of the equation, primary care physicians find it increasingly difficult to maintain a busy and efficient office practice and provide timely care to hospitalized patients.

Nonetheless, many primary care physicians have decried the growth of hospitalism as an encroachment on their traditional turf. Most vocal have been family physicians, who have watched the erosion of their opportunities to provide other types of services such as obstetrics and surgery. ${ }^{4,5}$ With increasing numbers of physicians, competition develops for the more remunerative clinical activities. Increased competition diminishes each physician's opportunity to do these activities, which then leads to loss of credentials, restricted hospital privileges, and higher malpracticeinsurance rates. The present generation of young family physicians is leaving training with only limited experience in some of these activities. This change may make sense for those who intend to practice in settings where specialists abound, but not in more isolated locations.

Could hospitalism be the harbinger of a trend toward more encroachment by traditional, organ-centric, medical specialities into the practice domain of general internal medicine? It's not likely. General internists are far more oriented to providing cognitive services than they are to doing procedures. In addition, many procedures typically performed by general internists are relatively basic ones that can readily be handled in the office and often are not subject to credentialing. Hospitalism, however, may be the harbinger of a more pernicious trend-specialization by physical location.

Based on economic efficiencies, rather than any strong scientific rationale, many medical care organizations are creating geographically distinct delivery systems for targeted groups such as patients with diabetes or patients who are women. Given the current economic forces in health care, creation of these "carve outs" is apt to accelerate. As a result, there will be proposals for new specialty credentials and new boards to define and give out the credentials. To the extent that these calls for new specialties simply represent attempts to defend economic turf, they must be resisted vigorously. Furthermore, the setting where a service is delivered can change rapidly with technologic advances. Even without newly created specialities, however, many generalists will face difficult choices about the location and scope of their practices.

On the positive side, this process represents another step in the continuing evolution of the definition of practice for general internists and other primary care physicians. The underlying trend is going to be greater opportunities for individualizing the scope of practice. In some respects, this trend also reflects a return to the prespecialization era, when all internists were generalists although many cultivated areas of special interest or expertise. The availability of special credentials for specific procedures or knowledge areas, such as those currently being developed by the Institute for Clinical Evaluation (http://www.abim.org/ice/ default.htm) for electrocardiography, should assist generalist physicians in obtaining and maintaining special expertise and skills. Other topic areas such as dermatology and women's health probably will follow. These credentials do not require a prescribed training program but simply the demonstration of clearly defined competencies. This opportunity will enable each practicing physician to formulate an individual approach to study.

In this issue Bryant promotes the concept of customizing practice. ${ }^{6}$ He explores the menu of opportunities available to general internists who elect (or are forced) to forgo management of hospitalized patients and proposes substitute activities. These substitutes range from doing clinical procedures to teaching and educational activities.

Dr. Bryant proposes the moniker "officist" for the outpatient physician. This label should be resisted not only because it is unwieldy but also because it promotes specialization by physical location rather than by content of practice. As Bryant contends, the practice of internal medicine is defined by an overarching body of knowledge and a set of professional attitudes rather than possession of specific skills.

At the core, physicians whose practices satisfy the American College of Physicians' definition of general internal 
medicine should retain that identity, for it best describes our work, our philosophy, and our future.-STEPHAN D. Finn, MD, MPH, VA Puget Sound Health Care System, University of Washington, Seattle, Wash.

\section{REFERENCES}

1. Wachter RM, Goldman L. The emerging role of "hospitalists" in the American health care system. N Engl J Med. 1996;335:514-7.

2. Diamond HS, Goldberg E, Janosky JE. The effect of full-time fac- ulty hospitalists on the efficiency of care at a community teaching hospital. Ann Intern Med. 1998;129:197-203.

3. Gipe B. A Pennsylvania model for in-house acute care physician services: improving inpatient performance and relieving outpatient stress. Cost Quality. 1996;2:6.

4. Brown RG. Hospitalist concept: another dangerous trend. Am Fam Physician. 1998;58:339-40.

5. McConaghy JR. The emerging role of hospitalists: will family physcians continue to practice hospital medicine? J Am Board Fam Pract. 1998;11:324-6.

6. Bryant DC. Hospitalist and 'officists': preparing for the future of general internal medicine. J Gen Intern Med. 1999; 14:182-6.

\begin{tabular}{|c||}
\hline ANNOUNCEMENT \\
SGIM Website \\
Please visit the Society of General Internal \\
Medicine on their World-Wide Website. \\
SGIM is located at \\
http://www.sgim.org
\end{tabular}

